\title{
Síndrome de takotsubo ¿Qué hay de nuevo?
}

\author{
Jessica Garduño-López ${ }^{1 *}$, Miguel Ángel Hernández-Márquez ${ }^{1}$ Emmanuel Adrián Lazcano-Díaz ${ }^{2}$ y \\ Francisco Martín Baranda-Tovar ${ }^{3}$

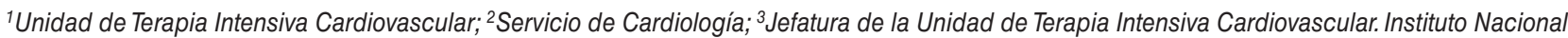 \\ de Cardiología Ignacio Chávez, Ciudad de México, México
}

\section{Resumen}

El síndrome de takotsubo (STT) es una entidad importante que simula un infarto agudo de miocardio. Existen cuatro variantes anatómicas descritas de esta entidad, importantes de reconocer. Ocurre mayormente en mujeres posmenopáusicas, generalmente se caracteriza por una hipocinesia transitoria del ápex del ventrículo izquierdo, los cuales resuelven en días a semanas, y se asocia con estrés emocional o físico. Su pronóstico es generalmente bueno. Sin embargo, pueden existir complicaciones graves como estado de choque por insuficiencia cardíaca, rotura ventricular o torsade de pointes. La fisiopatología no está completamente clara hasta el momento, sin embargo se ha sugerido que el espasmo coronario, la disfunción microvascular coronaria, la segregación catecolaminérgica, la miocarditis y el déficit de estrógenos podrían contribuir a la patogénesis del STT. Existen criterios internacionales (InterTAK Diagnostic Criteria) utilizados para el diagnóstico del STT. Debido a que los pacientes con STT padecen isquemia miocárdica grave y todos los criterios de síndromes coronarios agudos, este debe incluirse de manera más apropiada dentro del espectro de la cardiopatía isquémica.

Palabras clave: Cardiomiopatía. Catecolaminas. Insuficiencia cardíaca. Infarto de miocardio. Estrés. México.

\section{Takotsubo syndrome. What's new?}

\begin{abstract}
Takotsubo syndrome (STT) is an important entity that simulates an acute myocardial infarction. There are four described anatomical variants of this entity, important to recognize. It occurs mostly in postmenopausal women, is usually characterized by transient hypokinesia of the apex of the left ventricle, which resolves in days to weeks, and is associated with emotional or physical stress. The prognosis is generally good. However, there may be serious complications such as cardiogenic shock, heart failure, ventricular rupture, torsade de pointes. The physiopathology is not completely known up to now. However, it has been suggested that coronary spasm, coronary microvascular dysfunction, catecholaminergic segregation, myocarditis, and estrogen deficit could contribute to the pathogenesis of STT. There are international criteria (InterTAK Diagnostic Criteria) used for the diagnosis of STT. Because patients with takotsubo syndrome suffer from severe myocardial ischemia and all criteria for acute coronary syndromes, this should be included more appropriately within the spectrum of ischemic heart disease.
\end{abstract}

Key words: Cardiomyopathy. Catecholamine. Heart failure. Myocardial infarction. Stress. Mexico.

\section{Correspondencia:}

*Jessica Garduño-López

Belisario Domínguez, 1

Col. Sección XVI, Del. Tlalpan

Fecha de recepción: 06-02-2019

Fecha de aceptación: 07-03-2019

DOI: $10.24875 /$ REIE. 19000013
Disponible en internet: 20-06-2019 Rev Educ Investig Emer. 2019;1(2):54-65 www.medicinadeemergencias.com 


\section{Introducción}

El síndrome de takotsubo (STT) es una anomalía del movimiento de la pared (más frecuentemente apical) del ventrículo izquierdo (VI) de aparición aguda y usualmente reversible que se presenta tras situaciones de estrés físico y/o emocional. Se sugieren varios mecanismos fisiopatológicos involucrados, como la miocarditis, el espasmo de la arteria coronaria o la miocitolisis focal debida al aumento de catecolaminas ${ }^{1,2}$.

En 2006, la American Heart Association incorporó este trastorno en la clasificación de las miocardiopatías como una miocardiopatía adquirida ${ }^{3}$. En la actualidad sigue siendo considerada como una miocardiopatía, sin embargo se ha propuesto incluirla como parte del "síndrome de infarto agudo de miocardio sin ateroesclerosis coronaria obstructiva», ya que los pacientes con esta afección padecen isquemia miocárdica grave y cumplen todos los criterios de los síndromes coronarios agudos ${ }^{4,5}$.

En esta revisión resumiremos las teorías fisiopatológicas, clasificación y variantes anatómicas actuales, así como los criterios internacionales de diagnóstico y finalmente se realizarán comentarios actuales sobre el tratamiento.

\section{Historia}

EI STT ha recibido diferentes nombres desde sus primeras descripciones a principios de la década de 1990: síndrome del corazón roto, miocardio aturdido neurogénico, cardiomiopatía de estrés, discinesia apical transitoria, cardiomiopatía transitoria o inflado apical y cardiomiopatía de takotsubo ${ }^{6}$.

El término takotsubo (tako = pulpo, tsubo = olla) hace referencia a una olla con fondo redondo y cuello estrecho que se usa para atrapar pulpos en Japón, y que se asemeja al ventriculograma izquierdo durante la sístole en el STT ${ }^{2}$. Fue introducido por Sato y Dote en 1990 y 1991 para describir la semejanza de la circunferencia anatómica del VI durante la sístole en 5 pacientes con características clínicas de infarto de miocardio (IM), pero sin evidencia de obstrucción coronaria ${ }^{1,7}$.

\section{Epidemiología}

Se estima que la incidencia de STT es de alrededor del $2 \%$ de todos los pacientes con troponina positiva, un 1-3\% con elevación del segmento ST que presentan sospecha de síndrome isquémico coronario agudo (SICA) y en mujeres puede presentarse hasta en un $5-6 \% 8,9$.

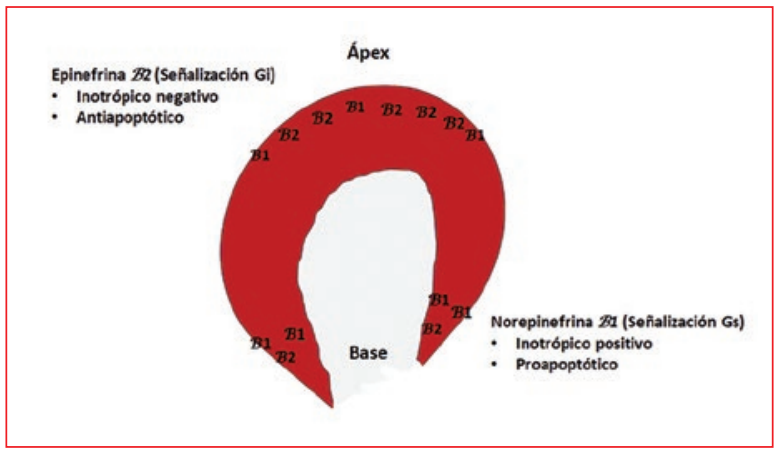

Figura 1. Representación esquemática de la inervación simpática de la base y ápex cardíaca (adaptada de Lyon, et al., 200812).

En EE.UU. se calcula que representa un $0.02 \%$ de las hospitalizaciones. Hasta un $90 \%$ son mujeres, con edad media de 67-70 años, y alrededor del $80 \%$ son mayores de 50 años. Las mujeres mayores de 55 años tienen 5 veces más probabilidades de presentarlo. Datos actuales refieren que la presentación en afroamericanos e hispanos es menos frecuente y más común en caucásicos; sin embargo se ha reportado que los afroamericanos presentan mayores complicaciones. La incidencia de la variante invertida de STT (STTr) es variable en la literatura, desde el 2.2 al 23\%,

\section{Etiología y fisiopatología \\ Activación e hiperactividad simpática}

La característica común en los casos primarios y secundarios de STT es el aumento de catecolaminas y de actividad simpática. Se han demostrado niveles extremadamente altos de catecolaminas en estos pacientes generados por un evento emocionalmente estresante, administración de epinefrina u otros simpaticomiméticos. En el locus coeruleus, los impulsos neurales descienden hacia el hipotálamo posterior, la vía de la activación simpática. Desde aquí, las vías simpáticas descienden a través de la médula espinal craneal y sacra y desencadenan la liberación de norepinefrina.La inervación cardíaca simpática se origina principalmente en los ganglios estrellados derecho e izquierdo. El mecanismo de cardiotoxicidad generado por las catecolaminas genera un aumento de la función de los receptores adrenérgicos incrementando la frecuencia cardíaca y la contractilidad cardíaca con un desequilibrio secundario en la relación entre el suministro y la demanda de oxígeno, creando áreas de hipoxia miocelular ${ }^{11,12}$ (Fig. 1). 


\section{Cambios metabólicos y electrolíticos}

La hipoxia miocítica puede agravarse aún más por el desacoplamiento de la fosforilación oxidativa en las mitocondrias, lo que inhibe el acoplamiento entre el transporte de electrones y las reacciones de fosforilación, que a su vez interferirán con la síntesis de ATP. Los cambios en la permeabilidad de la membrana también pueden provocar cambios en los electrolitos. Estos incluyen hipopotasemia, hipocalcemia e hipomagnesemia, con elevaciones resultantes en la hormona paratiroidea, disminución de zinc con hiposelenemia, que son defensas antioxidantes. La homeostasis catiónica alterada podría afectar varios procesos celulares y contribuir a la toxicidad miocárdica. La norepinefrina y la epinefrina también son fuentes potenciales de radicales libres. Estos radicales libres derivados del oxígeno pueden interferir con los transportadores de calcio y sodio, lo que puede resultar en una disfunción adicional de los miocitos. El desequilibrio entre el nivel de perfusión miocárdica y el metabolismo miocárdico que se encuentra comprometido y la etiología de este desequilibrio pueden asociarse a la resistencia a la insulina y los altos niveles de catecolaminas circulantes ${ }^{11,13}$.

\section{Incremento de catecolaminas circulantes}

Las catecolaminas liberadas directamente en el miocardio a través de los nervios simpáticos sugieren un efecto «tóxico» mayor que el que llega al corazón a través del torrente sanguíneo. De hecho, la diseminación de norepinefrina desde las terminales nerviosas simpáticas puede disminuir la viabilidad de los miocitos por medio de la sobrecarga de calcio mediada por adenosín monofosfato cíclico (AMPc), lo que resulta, histológicamente, en necrosis de la banda de contracción, que es una de las características patológicas del STT, junto con el aumento de la producción de matriz extracelular, lo que lleva a un rápido aumento de la fibrosis y la infiltración de neutrófilos. Sin embargo, el miocardio afectado representa un alto potencial de reconstitución estructural que se correlaciona con la rápida recuperación ${ }^{14}$.

El feocromocitoma conduce a cambios degenerativos en las fibras musculares, focos de necrosis, inflamación aguda, exudación inflamatoria intersticial crónica y fibrosis reparadora. Existe mayor daño miocárdico probablemente debido a la exposición persistente de los pacientes a niveles elevados de catecolamina. En el STT la elevación es transitoria y generalmente resulta en un daño menos evidente, como lo demuestra la elevación relativamente leve de los biomarcadores de necrosis ${ }^{15,16}$.

\section{Disfunción endotelial y la deficiencia de estrógenos}

Existe la posibilidad de que la disfunción endotelial pueda constituir un vínculo crucial entre una oleada simpática, lo que podría explicar la propensión al espasmo coronario epicárdico y/o microvascular y la isquemia miocárdica en el STT'17.

Este desequilibrio entre los factores vasoconstrictores y vasodilatadores puede generar una asociación entre el estrés, el péptido natriurético y la proteína de choque térmico. Además se sugiere que la deficiencia de estrógenos después de la menopausia podría facilitar la aparición de STT, ya sea por acción indirecta sobre el sistema nervioso o por acción directa sobre el corazón. Además, la mayoría de los casos de STT se presentan en pacientes con diversas comorbilidades neurológicas, psiquiátricas, pulmonares, renales, hepáticas y del tejido conectivo, que se asocian con disfunción endotelial ${ }^{18}$.

\section{Espasmo microvascular}

Otro mecanismo patogénico vascular que podría estar involucrado en el STT es la isquemia miocárdica aguda y transitoria. De hecho, desde las primeras publicaciones se ha sugerido que el vasoespasmo coronario es un factor causal. La estrecha relación entre la mejoría de la perfusión miocárdica y la disfunción del VI sugiere un papel patogénico de la disfunción microvascular coronaria ${ }^{19}$. El vasoespasmo epicárdico puede desencadenar la aparición del STT, pero no se considera que sea el principal mecanismo debido a que el vasoespasmo espontáneo rara vez se informa (5-10\%); las características histopatológicas de las muestras de biopsia endomiocárdica obtenidas de pacientes con STT muestran patrones de anomalías miocárdicas no asociadas con el miocardio infartado o en hibernación, y el área de la pared ventricular izquierda con disfunción del movimiento en el STT es inconsistente con la distribución arterial coronaria ${ }^{20}$.

Varios estudios de perfusión por tomografía computarizada por emisión de fotón único (SPECT) han mostrado una disminución en la captación del marcador durante la fase aguda del STT y un retorno a la normalidad en el seguimiento, lo que sugiere un papel para la disfunción microvascular coronaria como un 


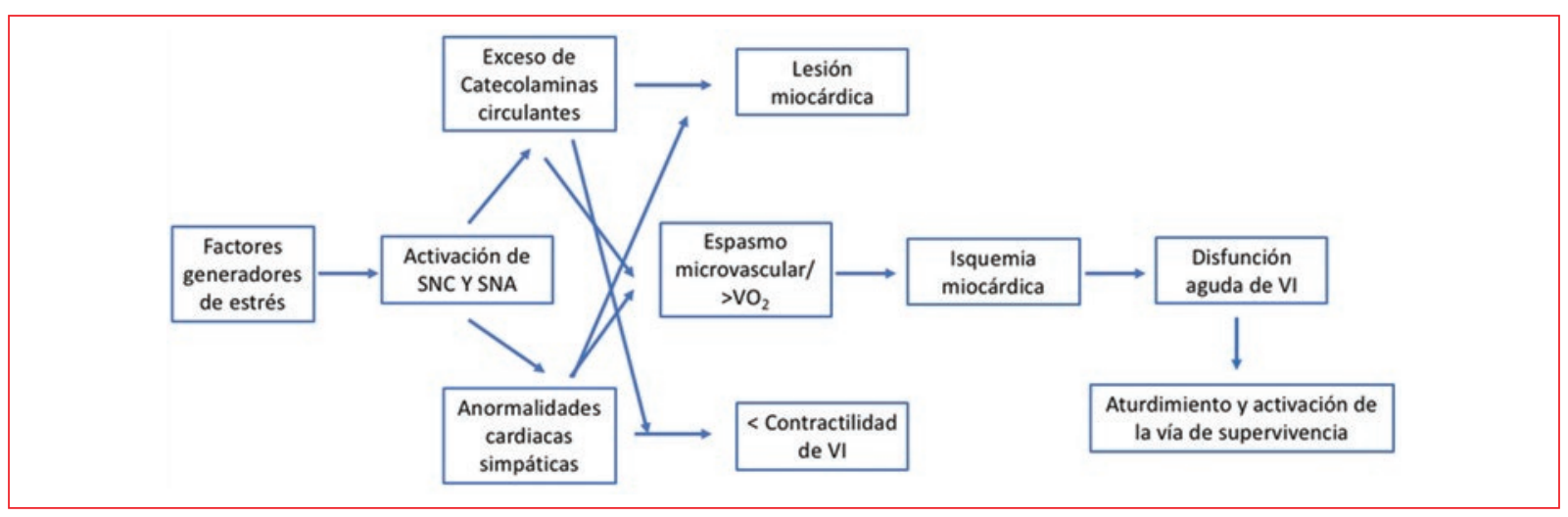

Figura 2. Fisiopatología del síndrome de takotsubo. Interacción entre factores desencadenantes y mecanismos de lesión cardíaca (adaptada de Pelliccia, et al., 2017¹1).

SNC: sistema nervioso central; SNA: sistema nervioso autónomo; VI: ventrículo izquierdo; $\mathrm{VO}_{2}$ : consumo de oxígeno en el miocardio.

desencadenante de isquemia miocárdica en esta condición ${ }^{21}$.

Actualmente se propone que el aumento de la concentración de catecolaminas en la fase aguda del STT induce lesión miocárdica directa y vasoconstricción coronaria, principalmente a nivel microvascular, junto con un aumento de la carga de trabajo cardíaco que contribuye a una situación de desajuste en la demanda seguido de aturdimiento postisquémico, seguido de una recuperación funcional completa en la mayoría de los casos. EI STT es una nueva forma de cardiopatía isquémica11 (Fig. 2).

\section{Presentación clínica, signos y síntomas}

Los síntomas más comunes del STT son dolor torácico de características anginosas, palpitaciones, disnea o síncope, precedidos por un evento emocional o físicamente estresante. Los síntomas adicionales incluyen náuseas, dolor abdominal, diaforesis, dolor epigástrico e indigestión. Estos pacientes también presentan con frecuencia insuficiencia cardíaca congestiva leve a moderada representada por una fracción de expulsión del VI disminuida. Pueden tener hipotensión debido a la reducción del volumen sistólico o la obstrucción del flujo de salida del VI. En el International Takotsubo Registry, los pacientes con STTr presentaron con más frecuencia un trastorno neurológico, mientras que los pacientes con STT presentaron un episodio psiquiátrico agudo ${ }^{22}$. EI STT puede diagnosticarse incidentalmente por nuevos cambios en el electrocardiograma (ECG) o una elevación repentina de biomarcadores cardíacos.
En cuanto a las complicaciones más frecuentemente vistas se encuentran: insuficiencia cardíaca (12-45\%), obstrucción del tracto de salida del ventrículo izquierdo (OTSVI) (10-25\%), insuficiencia mitral (14-25\%), choque cardiogénico (6-20\%), fibrilación auricular (5-15\%), trombo en VI (2-8\%), paro cardíaco (4-6\%), bloqueo auriculoventricular $(5 \%)$, taquiarritmias $(2-5 \%)$, bradiarritmias (2-5\%), torsade de pointes (2-5\%), muerte (1$4.5 \%)$, taquicardia o fibrilación ventricular (3\%) y defecto agudo del tabique ventricular $(<1 \%)^{23}$.

\section{Subtipos clínicos}

\section{Síndrome de takotsubo primario}

Los síntomas cardíacos agudos son el motivo de atención en las áreas de urgencias. Estos pacientes pueden presentar o no factores estresantes identificables, una gran mayoría de tipo emocionales. Pueden tener factores de riesgo predisponentes, pero no son la causa principal del aumento de catecolaminas. Su tratamiento clínico depende de las complicaciones primarias específicas ${ }^{11,24}$.

\section{Síndrome de takotsubo secundario}

Generalmente ocurre en pacientes ya hospitalizados por otra afección médica, quirúrgica, anestésica, obstétrica o psiquiátrica (Tabla 1). Se genera una activación repentina del sistema nervioso simpático y el aumento de catecolaminas lo precipita como una complicación de la condición primaria o su tratamiento. Su tratamiento debe centrarse no solo en sus 
Tabla 1. Desencadenantes del síndrome de takotsubo secundario

Desencadenantes del síndrome de takotsubo secundario

\section{Endocrino}

Feocromocitoma, tirotoxicosis, SIADH, crisis addisoniana, estado hiperosmolar hiperglucémico, coma mixedematoso, deficiencia de hormona adenocorticotrópica

Neurológico/neuroquirúrgico

Hemorragia subaracnoidea, traumatismo craneoencefálico, lesión medular, evento vascular cerebral, crisis convulsivas, miastenia gravis, síndrome de Guillain Barré Respiratorio
EPOC exacerbado, tromboembolia pulmonar, neumotórax a
tensión

Obstétrico

Aborto involuntario, labor de parto, cesárea de emergencia

Psiquiátrico

Ataque de ansiedad, ataque de pánico, intento suicida, síndrome de abstinencia, terapia electroconvulsiva

\section{Gastrointestinal}

Colecistitis aguda, cólico biliar, pancreatitis aguda, hiperemesis, diarrea severa, colitis pseudomembranosa, peritonitis

\section{Cardiovascular}

Ecocardiografía con dobutamina estrés, ablación de arritmias con radiofrecuencia, colocación de marcapasos, cardioversión eléctrica, fibrilación ventricular, arresto cardíaco

Hematológico

Transfusión sanguínea, púrpura trombótica trombocitopénica

Cirugía

Inducción anestésica

Medicamentos y drogas de abuso

Epinefrina, nortriptilina, venlafaxina, albuterol, flecainida, duloxetina, cocaína

Infección/sepsis

SIADH: síndrome de secreción inapropiada de hormona antidiurética; EPOC: enfermedad pulmonar obstructiva crónica. Adaptado de Lyon, et al., $2015^{25}$.

complicaciones cardíacas, sino también en la afección que desencadenó el síndrome. Es importante reconocer que después de cualquier tipo de cirugía, especialmente cirugía cardíaca, debe sospecharse el STT si se cumplen los criterios clínicos e instrumentales, y se diferencia rápidamente del IM, más frecuente. El pronóstico puede ser favorable si se inicia rápidamente tratamiento médico adecuado $0^{10,24,25}$.

\section{Variantes anatómicas}

La disfunción del VI en el STT incluye no solo la forma clásica de balloning apical, sino también diferentes patrones angiográficos que se han informado cada vez más a lo largo de la última década. Entre los 1,750 pacientes de un estudio, el tipo más común de miocardiopatía takotsubo fue el tipo apical (81.7\%), seguido del tipo ventricular medio (14.6\%), que respeta tanto el vértice como la base, el tipo basal o «takotsubo invertido", en el que hay anomalías en el movimiento de la pared basal y los segmentos medios con movimiento preservado o hipercinético del ápice, es muy raro (2.2\%), y el tipo focal $(1.5 \%)^{8}$ (Fig. 3). Algunos reportes de casos han documentado anomalías simultáneas en los ventrículos izquierdo y derecho hasta en un tercio de los casos pero la afectación aislada del ventrículo derecho (VD) es muy poco frecuente ${ }^{26}$. Recientemente, se describió el primer caso de "doble takotsubo", en el que el patrón típico fue seguido por el tipo invertido ${ }^{27}$. Por lo tanto, el STT puede afectar diferentes paredes miocárdicas, pero debemos recordar que en cualquier caso, esto se extiende más allá del territorio de una sola arteria coronaria epicárdica ${ }^{4}$.

\section{Diagnóstico}

\section{Auxiliares diagnósticos}

\section{ELECTROCARDIOGRAMA}

El ECG es esencial en todo paciente con dolor precordial y es anormal en la mayoría de los pacientes con STT en fase aguda (más del 95\%) ${ }^{23,25,28}$; muestra alteraciones que frecuentemente simulan un IM. En el International Takotsubo Registry se observó elevación del ST en el $44 \%$, inversión del ST en el $8 \%$, inversión de la onda $T$ en el $41 \%$ y bloqueo de rama izquierda en el 5\% ${ }^{8,23}$. La evolución típica es la elevación del segmento ST (a veces nuevo bloqueo de rama izquierda o menos frecuentemente inversión del segmento ST) dentro de las primeras $12 \mathrm{~h}$, seguido de aparición de ondas $\mathrm{Q}$ e inversión amplia y profunda de la onda $T$ con prolongación del intervalo QTC en las siguientes 24-48 $h^{25,29}$. Cuando la presentación es tardía pueden observarse inversiones gigantes de la onda T con prolongación del intervalo QTC 


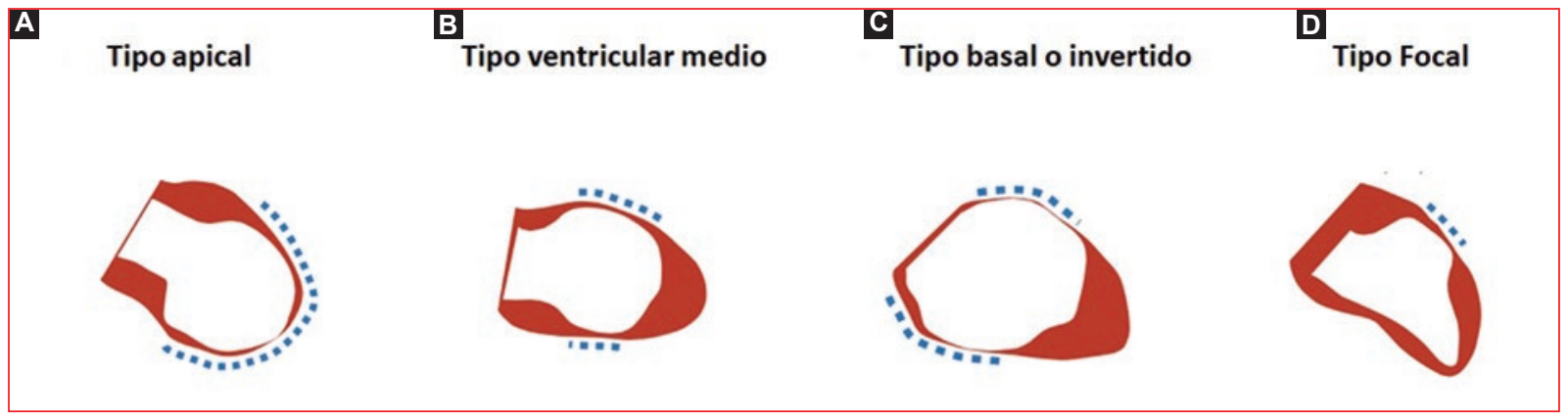

Figura 3. Cuatro variantes anatómicas de la miocardiopatía de takotsubo en orden de presentación. A: tipo apical $(81.7 \%)$. B: tipo ventricular medio (14.6\%). C: tipo basal o invertido (2.2\%). D: tipo focal (1.5\%) (adaptada de Templin, et al., 2015 $)$.

sin elevación del segmento $\mathrm{ST}^{25,28}$. La prolongación del QTc a menudo es pronunciada (> $500 \mathrm{~ms}$ ), predisponiendo a torsade de pointes y fibrilación ventricular ${ }^{25}$. Mientras que en el IM anterior la elevación del ST suele observarse en V1-V4, I y aVL, en el STT suele centrarse en V2-V5, II y aVR (esta última derivación alineada con el ápex) ${ }^{23}$. Pese a que el ECG no es suficiente para el diagnóstico y ningún hallazgo, solo o en combinación, debe retrasar $\mathrm{u}$ obviar el cateterismo coronario ${ }^{30}$, se han propuesto criterios electrocardiográficos que mejoran la precisión de este método, como la combinación de elevación del segmento ST en aVR con elevación del ST en derivaciones anteroseptales, que obtuvieron una especificidad y valor predictivo positivo del $100 \%$ ( $p<$ $0.001)^{31}$. La elevación del ST en cara inferior es infrecuente en el STT ${ }^{23}$.

\section{BIOMARCADORES}

La práctica totalidad de los casos de STT exhiben evidencia de necrosis miocárdica ${ }^{23}$, evidenciada por elevación de los niveles de Creatincinasa (CK), Creatincinasa-MB (CK-MB) y troponinas ${ }^{32}$. Los niveles iniciales de troponinas son iguales que en aquellos con IM, pero el pico máximo suele ser considerablemente menor en el STT ${ }^{23}$. Además, los niveles de estos biomarcadores son desproporcionadamente bajos comparados con las alteraciones de la movilidad parietal presentadas, lo que quizá ponga en evidencia un miocardio reversiblemente aturdido $23,28,29,32$. Las troponinas I y T, medidas por métodos convencionales, están elevadas en $>90 \%$ de los pacientes y los niveles máximos generalmente son < $10 \mathrm{ng} / \mathrm{ml}^{25,28}$. Niveles más altos son predictores de mal pronóstico intrahospitalario ${ }^{23}$.

Los péptidos natriuréticos péptido natriurético cerebral (BNP) y $\mathrm{N}$ terminal del propéptido natriurético cerebral
(NT-proBNP) están casi siempre elevados, correlacionan con la extensión de las anormalidades de la movilidad parieta ${ }^{28}$ y en niveles mayores que en pacientes con $\mathrm{IM}^{28,33}$, alcanzan su máximo a las $48 \mathrm{~h}$ y se mantienen hasta 3 meses $^{28}$. Además, sus niveles parecen correlacionar con el grado de sobreactivación simpática y la concentración pico de proteína $\mathrm{C}$ reactiva ${ }^{23}$. Este comportamiento sugiere que estos péptidos son mejores marcadores que las troponinas y deberían medirse en todo paciente con sospecha de STT ${ }^{25}$; el cociente BNP/ troponina podría ayudar a diferenciar entre STT e $\mathrm{IM}^{28,33}$.

Otros biomarcadores prometedores incluyen los microRNA (miRNA). Jaguszewski ${ }^{34}$ ha identificado una firma de 4 miRNA circulantes (miR-16, miR26a, miR-1 y miR-133a) relacionados con la respuesta al estrés como novedosos biomarcadores para diferenciar entre STT e IM, evidenciando además una fisiopatología distinta ${ }^{25}$ y perfilándose como futuras opciones diagnósticas ${ }^{32}$. Otras sustancias, como las catecolaminas, el neuropéptido $Y$ o la serotonina, que se muestran elevadas en el STT ${ }^{25}$, no han encontrado el camino para convertirse en herramientas útiles en la práctica.

\section{IMAGEN INVASIVA}

\section{Coronariografía invasiva y ventriculografía}

Casi todos los pacientes con sospecha de STT y todos aquellos que presentan elevación del segmento ST deben ir a coronariografía invasiva ${ }^{23,32}$, siendo este el mejor método para diferenciar al STT de un SICA ${ }^{25,32}$. En la mayoría de los pacientes con STT las arterias coronarias son normales y sin obstrucciones, no obstante lo cual, en la actualidad está ampliamente aceptado que el STT puede coexistir con enfermedad arterial coronaria aterosclerótica (prevalencia del 10-61\%, 
con $15.3 \%$ en el International Takotsubo Registry) ${ }^{29,35}$, en especial cuando se trata de pacientes más ancianos con factores de riesgo ${ }^{25,28}$. En este caso, será importante determinar si la enfermedad arterial coronaria es suficiente para explicar el grado o el patrón de disfunción ventricular ${ }^{25}$. Las técnicas de imagen intracoronaria, tales como la tomografía de coherencia óptica han sido útiles para el descarte definitivo de anormalidades estructurales en los vasos epicárdicos que podrían pasar desapercibidas en la angiografía, como rotura de placa, erosión de la íntima, disecciones o trombo residual, lo que apoyaría la teoría de una explicación fisiopatológica distinta ${ }^{5}$. Junto a la coronariografía debe realizarse una ventriculografía izquierda que incluya mediciones de presión (presión telediastólica del VI), útil en la correcta clasificación del tipo de STT. La alteración regional de la movilidad parietal que se extiende más allá del territorio de una única arteria coronaria es una de las características más importantes para distinguir el STT de la enfermedad arterial coronaria ${ }^{32}$. Finalmente, si un paciente puede tener simultáneamente STT y SICA es motivo de debate, pero dado que este último es motivo de estrés físico y emocional, la posibilidad debe siempre tenerse en cuenta ${ }^{28}$.

\section{IMAGEN NO INVASIVA}

\section{Ecocardiograma}

Es un estudio imperativo en el diagnóstico y curso del STT, ofrece rápida accesibilidad en la evaluación de la disfunción sistólica del $\mathrm{V}^{5,32}$ y debería ser el primer estudio de imagen no invasiva cuando se sospeche el diagnóstico ${ }^{25}$. Las características ecocardiográficas clave durante la fase aguda consisten en una gran área de miocardio disfuncional que se extiende más allá del territorio de una única arteria coronaria y usualmente se caracteriza por anormalidades regionales simétricas que involucran los segmentos medioventriculares de las paredes anterior, inferior y lateral, dando un patrón circunferencial ${ }^{25,28}$. Los hallazgos típicos del STT con balloning apical consisten en acinesia, hipocinesia o discinesia de los segmentos apicales y medios del VI e hipercinesia o normalidad de los segmentos basales ${ }^{33}$. El ecocardiograma permite además detectar complicaciones, como presencia de trombos intracavitarios, involucro del VD o movimiento sistólico anterior de la valva anterior de la mitral, que puede ser causa de insuficiencia mitral aguda o (junto con la hipercontractilidad de la base) ser causa de OTSVI ${ }^{5,28,32,36}$. Su uso es obligatorio en el seguimiento para confirmar la recuperación ${ }^{32}$ y cobra especial relevancia cuando las alteraciones de la movilidad revierten con rapidez, incluso en las primeras $48 \mathrm{~h}^{5}$.

\section{Resonancia magnética}

Es un estudio con difícil accesibilidad en la fase aguda, pero cobra gran relevancia en el periodo subagudo ${ }^{23}$. Permite visualizar con gran precisión las anormalidades de la movilidad regional tanto en el VI como en VD, lo que permite distinguir al STT de otras enfermedades $^{32}$. Del mismo modo, permite detectar lesiones reversibles (inflamación, edema) e irreversibles (necrosis, fibrosis) todo ello útil en el contexto del $\mathrm{STT}^{33}$. El edema de distribución difusa o transmural en imágenes ponderadas en T2 y la ausencia de realce tardío con gadolinio (RTG) son claves para distinguir el STT del IM ${ }^{25,36}$. EI RTG siempre está presente en mayor o menor medida en el IM y hasta en el $88 \%$ (con patrón parcheado) de los casos en la miocarditis, siendo un marcador de fibrosis. Incluso cuando el STT presenta RTG, este suele ser de menor intensidad ${ }^{28,33}$. La resonancia magnética puede ser superior al ecocardiograma en la evaluación del VD y de trombos apicales $^{28,32}$. En el STT, el área de edema normalmente coincide con las alteraciones de la movilidad parietal a diferencia de lo visto en el IM, donde la distribución del edema coincide con el territorio de la arteria coronaria epicárdica ${ }^{28}$. Así mismo, se ha encontrado que la ausencia de RTG se relaciona con ausencia de daño miocárdico irreversible y predice recuperación funcional ${ }^{33}$. Por tanto, la resonancia magnética debería considerarse en todo paciente con sospecha de STT en fase aguda, aunque dada su baja disponibilidad, podría limitarse a casos indeterminados o limítrofes, así mismo, puede utilizarse para el seguimiento de la recuperación de la función del $\mathrm{V}^{25}$.

\section{Angiotomografía coronaria}

Para aquellos pacientes cuyas comorbilidades impiden realizar una coronariografía invasiva (diátesis hemorrágica, sangrado intracraneal), la angiotomografía coronaria puede ser una alternativa apropiada ${ }^{23}$ para excluir lesiones coronarias culpables en pacientes seleccionados con datos clínicos y ecocardiográficos convincentes de $\mathrm{STT}^{28,33}$. El estudio tomográfico puede aprovecharse también para evaluar las alteraciones de la movilidad, aunque esto implica mayor tiempo de exposición a la radiación. También tiene un rol potencial en pacientes que se presentan tardíamente (> $48 \mathrm{~h}$ 
Tabla 2. Criterios internacionales de síndrome de takotsubo (STT) (InterTAK Diagnostic Criteria)

\section{Criterios internacionales de síndrome de takotsubo}

1. Pacientes con disfunción ventricular izquierda transitoria (hipocinesia, acinesia o discinesia) que se presenta como balloning apical 0 anormalidades de la motilidad parietal medio ventricular, basal o focal. Puede haber presencia de compromiso ventricular derecho. Además de estos patrones de motilidad parietal regional, pueden existir transiciones entre todos los tipos de disfunción. La anormalidad de la motilidad parietal regional generalmente se extiende más allá de una única distribución vascular epicárdica; no obstante, pueden existir casos raros donde haya presencia de lesión coronaria en el territorio miocárdico comprendido por la anormalidad parietal.

2. Un desencadenante emocional, físico o combinado puede preceder el evento de STT, aunque no de manera obligatoria.

3. La presencia de alteraciones neurológicas (p. ej., hemorragia subaracnoidea, accidente cerebrovascular/ataque isquémico transitorio o convulsiones), así como feocromocitoma, no excluyen el diagnóstico de STT.

4. Presencia de nuevas alteraciones electrocardiográficas (elevación o descenso del segmento ST, inversión de la onda T o prolongación del intervalo QTc); sin embargo puede haber casos sin presencia de alteraciones electrocardiográficas.

5. Los niveles de biomarcadores cardíacos (troponina y CK) se encuentran moderadamente elevados en la mayoría de los casos; es común el aumento del péptido natriurético cerebral.

6. La enfermedad coronaria significativa no es una contradicción en el STT.

7. Los pacientes no presentan evidencia de miocarditis infecciosa (se sugiere realizar imagen de resonancia magnética para descartar dicha patología).

8. Las mujeres posmenopáusicas son predominantemente afectadas.

Adaptado de Ghadri, et al., $2018^{10}$.

posteriores al dolor), que están estables y asintomáticos, así como en pacientes que desean ser evaluados retrospectivamente (semanas o meses después de cuadro clínico $)^{25}$.

\section{IMAGEN NUCLEAR}

La SPECT y la tomografía por emisión de positrones (PET) pueden ser estudios suplementarios en el STT para evaluar inervación miocárdica, metabolismo y flujo coronario $^{5,36}$. Estas técnicas se han usado sobre todo para estudiar la fisiopatología del STT ${ }^{31}$. Mediante PET se ha demostrado que el flujo sanguíneo en las regiones adelgazadas (ápex generalmente) está preservado ${ }^{23,32}$, mientras que las regiones normofuncionantes hay hiperperfusión. Tanto la SPECT (usando ácido ${ }^{123}$ - $\beta$-metil-yodofenil pentadecanoico, que refleja ácidos grasos) como la PET (usando ${ }^{128} \mathrm{~F}$-fluorodesoxiglucosa, que refleja consumo de glucosa) suelen mostrar actividad metabólica reducida en las regiones afectadas, mientras que la perfusión se mantiene normal ${ }^{23}$. Usando SPECT, el consumo miocárdico de ${ }^{123}$-metayodobencilguanidina radioisotopo ( ${ }^{123}$ I-MIBG) refleja la inervación simpática miocárdica. La ${ }^{123} \mathrm{MIBG}$ se mantiene reducida durante meses (denervación) mientras que la perfusión es normal, incluso cuando la contractilidad se ha restablecido, lo que es consistente con alteraciones de la actividad neuronal simpática ${ }^{23,32}$. Idealmente la SPECT con ${ }^{123} \mathrm{MIBG}$ debería combinarse con escintigrafía de perfusión miocárdica para descartar $\mathrm{IM}^{3}$. No obstante todo esto, el rol de la imagen nuclear en la etapa aguda del STT no está bien definido.

\section{Integración diagnóstica}

El diagnóstico de STT se basa en la demostración de una anormalidad de la movilidad parietal regional del VI más allá del territorio de una única arteria coronaria que es reversible y que se asocia a un estresor físico o emocional ${ }^{28}$. Hay múltiples criterios diagnósticos publicados, siendo los más usados probablemente los criterios modificados de la Clínica Mayo ${ }^{36}$, sin embargo, el avance continuo en el entendimiento del STT ha hecho necesarios otros métodos para la integración diagnóstica. Recientemente se publicó, en dos partes, el International Expert Consensus Document on Takotsubo Syndrome ${ }^{10,23}$, que ofrece criterios internacionales (InterTAK Diagnostic Criteria) (Tabla 2), así como un algoritmo donde quedan implícitos estos nuevos conocimientos, incluyendo el InterTAK Diagnostic Score.

El algoritmo reconoce como primera medida determinar si se trata de un paciente con elevación del segmento ST, en cuyo caso se realizará una angiografía invasiva en búsqueda de lesiones culpables que expliquen las alteraciones de la movilidad regional, confirmando un SICA, o de lo contrario buscar datos de miocarditis, en cuya ausencia se confirmaría el diagnóstico de STT. Para el caso de que el paciente se presente con síntomas sugestivos sin elevación del segmento ST, se procede con el InterTAK Diagnostic Score (Tabla 3), que permitirá diferenciar pacientes con baja o intermedia probabilidad de STT (< 70 puntos), que deberían ir a coronariografía invasiva, de aquellos con probabilidad alta (> 70 puntos), que dado su contexto pueden 


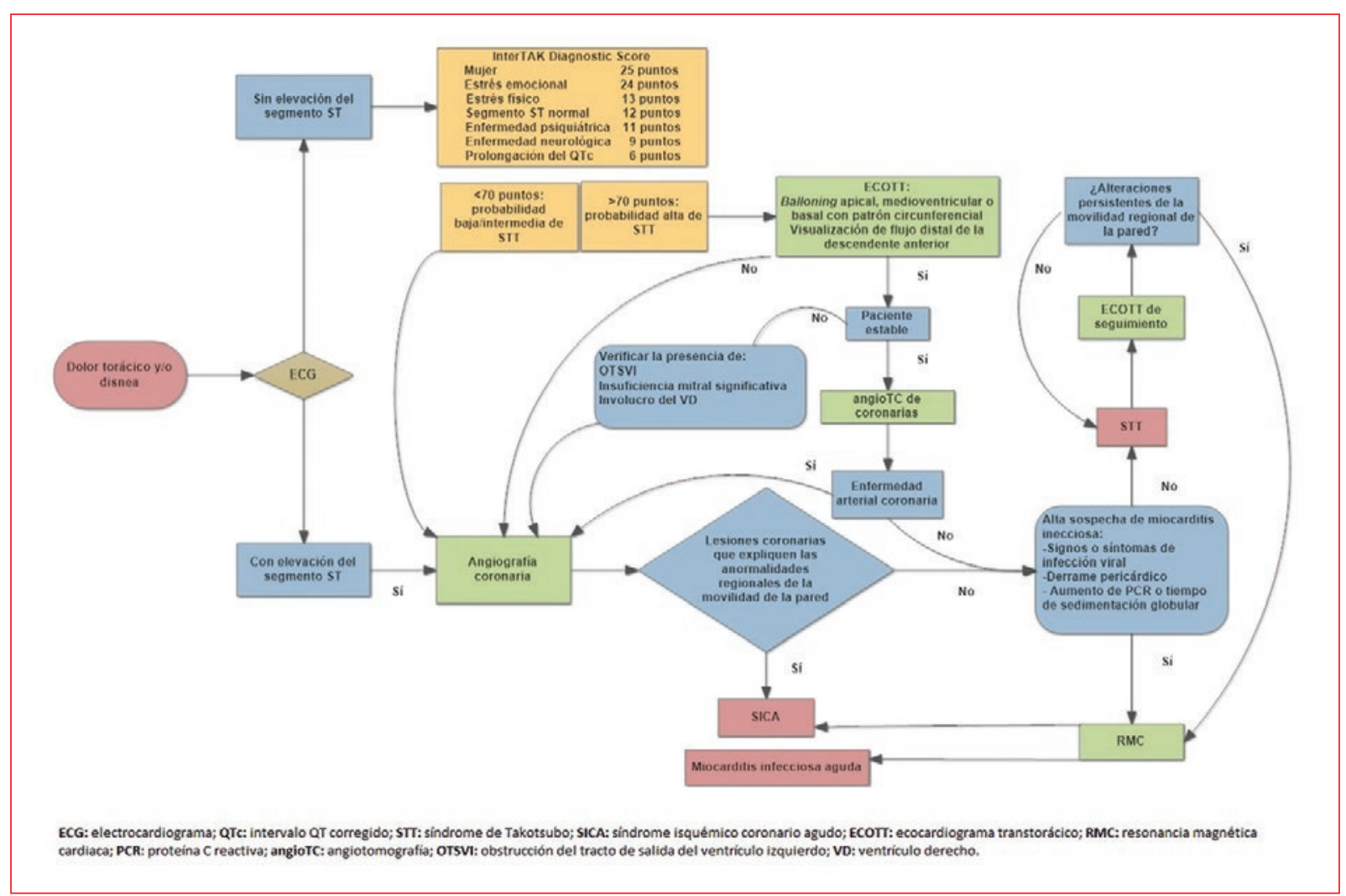

Figura 4. Algoritmo diagnóstico de Síndrome de TakoTsubo (elaboración propia).

Tabla 3. Escala de riesgo internacional (InterTAK Diagnostic Score). Pacientes con puntaje de 30 puntos tienen una probabilidad predicha $<1 \%$, mientras que los pacientes con 50 puntos tienen una probabilidad del $18 \%$ y pacientes con $>70$ puntos tienen probabilidad de aproximadamente un $90 \%$ de cursar con síndrome de takotsubo

\section{Criterio} Puntaje

Sexo femenino

25

Desencadenante emocional

Desencadenante físico 13

Ausencia de depresión del segmento ST (excepto en aVR)

Desordenes psiquiátricos

Desordenes neurológicos

Prolongación del QTC

aVR: Derivación unipolar aumentada de brazo derecho. Adaptado de Ghadri, et al., 201823.

evaluarse con ecocardiografía buscando alteraciones típicas que, en caso de estar presentes en un paciente estable, permiten dirigir la ruta diagnóstica hacia una angiotomografía de coronarias para búsqueda de enfermedad arterial coronaria. A partir de ahí, se decidirá si el paciente amerita ir a coronariografía invasiva, amerita resonancia magnética por sospecha de miocarditis o se confirma el diagnóstico de STT (Fig. 4) ${ }^{10}$.

\section{Tratamiento}

\section{Tratamiento en la fase aguda}

Muchos clínicos consideran ahora el STT como una enfermedad relevante, y aplican monitorización estricta y cuidados en la Unidad de pacientes críticos, dado el riesgo de arritmias (en especial en pacientes con prolongación del QTc) y otras complicaciones graves ${ }^{28,37}$. No existen guías de tratamiento para el STT, pues no hay ensayos clínicos dirigidos a estos pacientes ${ }^{28}$, de modo que el tratamiento se basa en la experiencia clínica y el consenso de expertos ${ }^{23}$. Como el cuadro inicial frecuentemente simula un IM, el tratamiento se basa en apoyo de oxígeno, ácido acetilsalicílico (AAS), heparina y morfina, aunque la eficacia de estos medicamentos no ha sido probada. Una vez que el diagnóstico de STT se establece, la estrategia terapéutica debería modificarse y dirigirse al tratamiento de sostén y la prevención y tratamiento de las complicaciones y de acuerdo con 
los factores de riesgo ${ }^{20,32}$. Para pacientes con congestión pulmonar y sin hipotensión o bajo gasto cardíaco el tratamiento se dirige a reducir el retorno venoso con vasodilatadores (nitroprusiato, nitroglicerina 0 nesiritida) y con diuréticos ${ }^{28}$. Los medicamentos que prolongan el QT deben usarse con precaución, pues pueden desencadenar taquicardia ventricular o torsade de pointes $^{23}$. En pacientes complicados con choque cardiogénico es importante detectar la existencia de OTSVI, en cuya presencia deberán suspenderse inmediatamente los inotrópicos (dobutamina, milrinona, dopamina), pues dicha obstrucción se asocia con la hipercontractilidad de la base; en estos pacientes, si no hay falla cardíaca severa, hipotensión o bradicardia, sería razonable el uso intravenoso de betabloqueantes de corta acción ${ }^{32}$. No obstante esto, los betabloqueantes no previenen el STT y no se sabe si los pacientes previamente tratados con estos fármacos tienen una evolución más benigna ${ }^{37,38}$. En el caso de que haya choque cardiogénico sin OTSVI, las catecolaminas (vasopresoras o inotrópicas) deberían usarse con precaución, dada su clara asociación fisiopatológica con el STT; en este sentido, el levosimendán puede considerarse como una opción "ahorradora de catecolaminas»32. El balón de contrapulsación intraaórtico se ha utilizado también, con poca evidencia, pero debe evitarse cuando hay OTSVI pues agrava el gradiente de presión $^{33}$. En pacientes con STT y choque refractario deben considerarse la membrana de oxigenación extracorpórea y los dispositivos de asistencia ventricular izquierda temporales ${ }^{32}$. El uso de desfibrilador/cardiovertor como prevención primaria o secundaria es incierto, dada la naturaleza reversible de las alteraciones en el VI y el ECG, y el riesgo residual de arritmias malignas luego de la recuperación es desconocido ${ }^{23}$.

\section{Tratamiento anticoagulante}

Aunque no hay ensayos clínicos en este apartado, debe considerarse dar anticoagulación formal a pacientes con grandes áreas de acinesia y continuarla hasta la recuperación de la función del VI. Para aquellos con formación de trombos, se recomienda la anticoagulación durante 3 meses $^{28}$.

\section{Tratamiento a largo plazo}

Los inhibidores de la enzima convertidora de angiotensina y los antagonistas del receptor de angiotensina se han asociado a mejoría en la supervivencia en el seguimiento a 1 año $^{32}$, así como disminución de las recurrencias, mientras que, pese a ser los medicamentos más indicados y tener una justificación fisiopatológica aparente, no hay ningún beneficio con el uso de betabloqueantes ${ }^{32,33}$. Para los pacientes con enfermedad arterial coronaria concomitante, el tratamiento con AAS y estatinas es apropiado ${ }^{39}$, mientras que el tratamiento con estrógenos en mujeres posmenopáusicas o con fármacos psiquiátricos aún es controvertido, pero podrían ser beneficiosos ${ }^{23,32}$.

\section{Pronóstico y recurrencias}

Por definición, la disfunción del VI en el STT es reversible, el involucro del VD es ocasional y las complicaciones serias y las recurrencias son infrecuentes ${ }^{5}$. No obstante esto, el pronóstico en fase aguda en el STT es peor de lo que se pensaba ${ }^{29}$, con tasas intrahospitalarias de choque, maniobras de resucitación y muerte comparables en pacientes de la misma edad y sexo con el $\mathrm{SICA}^{37}$, lo cual parece estar relacionado con el perfil del paciente (fragilidad y comorbilidades) ${ }^{5}$. Los predictores de peor pronóstico intrahospitalario incluyen desencadenantes físicos, enfermedad neurológica o psiquiátrica aguda, niveles altos de troponina y fracción de eyección baja al ingreso ${ }^{29}$. Un metaanálisis reciente mostró que la fibrilación atrial duplicaba el riesgo de muerte por todas las causas en el STT (OR: 2.19; IC 95\%: 1.57-3.06; $p$ < $0.001)^{40}$. Datos recientes de registros multicéntricos sugieren que el pronóstico a largo plazo es también más pobre de lo que se creía ${ }^{37}$. Pelliccia, et al. ${ }^{41}$ encontraron en un metaanálisis de 54 estudios una mortalidad intrahospitalaria del $1.8 \%$ y una tasa de mortalidad a largo plazo del 3.5\% por año, y en el análisis de regresión hallaron que la mortalidad total a largo plazo se asociaba significativamente con la mayor edad, estresor físico y la forma típica de balloning apical. Un estudio observacional de casos y controles ${ }^{42}$ que estudió a pacientes que habían tenido STT más de 12 meses atrás encontró que el $88 \%$ aún tenían síntomas compatibles con insuficiencia cardíaca y limitación cardíaca en las pruebas de ejercicio (consumo pico de oxígeno disminuido $24 \pm$ 1.3 vs. $31 \pm 1.3 \mathrm{ml} / \mathrm{kg} / \mathrm{min}, p<0.001$ ), así como índices de deformación cardíaca alterados (strain circunferencial apical disminuido $-16 \pm 1.0$ vs. $-23 \pm 1.5 \%, p<0.001$; strain longitudinal global $-17 \pm 1.0$ vs. $-20 \pm 1.0 \%$, $p<0.006$ ) entre otros, todo lo cual sugiere que los pacientes que padecieron STT desarrollan un fenotipo persistente, a largo plazo, de falla cardíaca.

Las recurrencias de STT son bajas, reportándose una recurrencia anual promedio del $2.9 \%$ en los primeros años, que disminuye un $1.3 \%$ por año posteriormente ${ }^{5}$. 
El segundo episodio ocurre de 3 meses a 10 años posteriores al primero y la predominancia de mujeres y la forma de balloning apical aplica para las recurrencias, aunque un mismo paciente puede presentarse con diferentes variantes anatómicas ${ }^{20}$. No hay evidencia que apoye algún tratamiento para evitar las recurrencias en la fase crónica ${ }^{29}$.

\section{Conclusiones}

- EI STT se manifiesta con alteraciones regionales de la movilidad características del VI y usualmente tiene un desencadenante físico o emocional, tratándose de una patología normalmente reversible.

- La fisiopatología es compleja e incluye alteraciones en la microvasculatura, espasmo coronario, segregación catecolaminérgica, que resulta en el desequilibrio entre el consumo y aporte de oxígeno, llevando a isquemia y disfunción ventricular.

- La miocarditis y el déficit de estrógenos podrían contribuir a la patogénesis del STT.

- Actualmente se reconoce la existencia de variantes que van más allá del típico balloning apical.

- Hoy día, existen criterios y una escala internacionales, así como un algoritmo actualizado para la integración diagnóstica en el paciente en el que se sospecha el STT.

- Contrario a las concepciones anteriores, actualmente reconocemos que no se requiere de un estresor desencadenante, que las lesiones ateroescleróticas coronarias no excluyen el diagnóstico y que el pronóstico en la fase aguda y a largo plazo es peor de lo que se conocía.

- No existe suficiente evidencia en el tratamiento farmacológico para el STT. El tratamiento se enfoca en el tratamiento de sostén de la fase aguda y las complicaciones. Así mismo, el uso de catecolaminérgicos debe ser usado con precaución y el apoyo inotrópico debe ser evaluado adecuadamente, en especial en pacientes con OTSVI.

- Debido a que los pacientes con STT padecen isquemia miocárdica grave y todos los criterios de síndromes coronarios agudos, este debe incluirse de manera más apropiada dentro del espectro de la cardiopatía isquémica.

\section{Financiación}

La presente investigación no ha recibido ayuda específica proveniente de agencias del sector público, sector comercial o entidades sin ánimo de lucro.

\section{Conflicto de intereses}

Los autores declaran no tener ningún conflicto de intereses.

\section{Bibliografía}

1. Kurisu S, Sato H. History of cardiology in the last 100 years: Japanese contribution to studies on Tako-tsubo like left ventricular dysfunction. Nihon Naika Gakkai Zasshi. 2002;91(3):849-52.

2. Kurisu S, Kihara Y. Tako-tsubo cardiomyopathy: Clinical presentation and underlying mechanism. J Cardiol. 2012;60(6):429-37.

3. Maron BJ, Towbin JA, Thiene G, Antzelevitch C, Corrado D, Arnett D, et al. Contemporary definitions and classification of the cardiomyopathies. Circulation. 2006;113(14):1807-16.

4. Pelliccia F, Sinagra G, Elliott P, Parodi G, Basso C, Camici PG. takotsubo is not a cardiomyopathy. Int J Cardiol. 2018 Mar 1;254:250-3.

5. Mejía-Rentería HD, Núñez-Gil IJ. takotsubo syndrome: Advances in the understanding and management of an enigmatic stress cardiomyopathy. World J Cardiol. 2016;8(7):413

6. Y-Hassan S, Tornvall P. Epidemiology, pathogenesis, and management of takotsubo syndrome. Clin Auton Res. 2017;28(1):53-65.

7. Dote K, Sato H, Tateishi H, Uchida T, Ishihara M. Myocardial stunning due to simultaneous multivessel coronary spasms: a review of 5 cases. J Cardiol. 1991;21(2):203-14.

8. Templin C, Ghadri JR, Diekmann J, Napp LC, Bataiosu DR, Jaguszewski M, et al. Clinical Features and Outcomes of takotsubo (Stress) Cardiomyopathy. N Engl J Med. 2015;373(10):929-38.

9. Awad HH, McNeal AR, Goyal H. Reverse takotsubo cardiomyopathy: a comprehensive review. Ann Transl Med. 2018;6(23):460.

10. Ghadri J-R, Wittstein IS, Prasad A, Sharkey S, Dote K, Akashi YJ, et al. International expert consensus document on takotsubo Syndrome (Part I): Clinical characteristics, diagnostic criteria, and pathophysiology. Eur Heart J. 2018;39(22):2032-46.

11. Pelliccia F, Kaski JC, Crea F, Camici PG. Pathophysiology of takotsubo Syndrome. Circulation. 2017;135(24):2426-41.

12. Lyon AR, Rees PSC, Prasad S, Poole-Wilson PA, Harding SE. Stress (takotsubo) cardiomyopathy: A novel pathophysiological hypothesis to explain catecholamine-induced acute myocardial stunning. Nat Clin Pract Cardiovasc Med. 2008;5(1):22-9.

13. Zhang X, Szeto C, Gao E, Tang M, Jin J, Fu Q, et al. Cardiotoxic and cardioprotective features of chronic $\beta$-adrenergic signaling. Circ Res. 2013;112(3):498-509.

14. Nef HM, Möllmann H, Kostin S, Troidl C, Voss S, Weber M, et al. Tako-Tsubo cardiomyopathy: intraindividual structural analysis in the acute phase and after functional recovery. Eur Heart J. 2007;28(20):2456-64.

15. Akashi YJ, Nef HM, Lyon AR. Epidemiology and pathophysiology of takotsubo syndrome. Nat Rev Cardiol. 2015;12(7):387-97.

16. Lenders JW, Eisenhofer G, Mannelli M, Pacak K. Phaeochromocytoma. Lancet. 2005;366(9486):665-75.

17. Naegele M, Flammer AJ, Enseleit F, Roas S, Frank M, Hirt A, et al. Endothelial function and sympathetic nervous system activity in patients with takotsubo syndrome. Int J Cardiol. 2016 Dec 1;224:226-230.

18. Pelliccia F, Greco C, Vitale C, Rosano G, Gaudio C, Kaski JC. takotsubo syndrome (stress cardiomyopathy): an intriguing clinical condition in search of its identity. Am J Med. 2014 Aug;127(8):699-704.

19. Galiuto L, De Caterina AR, Porfidia A, Paraggio L, Barchetta S, Locorotondo $\mathrm{G}$, et al. Reversible coronary microvascular dysfunction: a common pathogenetic mechanism in apical ballooning or Tako-Tsubo syndrome. Eur Heart J. 2010;31(11):1319-27.

20. Tanabe Y, Akashi YJ. Improving the understanding of takotsubo syndrome: consequences of diagnosis and treatment. Expert Rev Cardiovasc Ther. 2016;14(6):737-48.

21. Ito $K$, Sugihara $H$, Kinoshita $N$, Azuma A, Matsubara $H$. Assessment of takotsubo cardiomyopathy (transient left ventricular apical ballooning) using 99mTc-tetrofosmin, 123I-BMIPP, 123I-MIBG and 99mTc-PYP myocardial SPECT. Ann Nucl Med. 2005;19(6):435-45.

22. Ghadri JR, Cammann VL, Napp LC, Jurisic S, Diekmann J, Bataiosu DR et al. Differences in the clinical profile and outcomes of typical and atypical takotsubo syndrome: Data from the International Takotsubo Registry. JAMA Cardiol. 2016; Jun 1;1(3):335-40.

23. Ghadri JR, Wittstein IS, Prasad A, Sharkey S, Dote K, Akashi YJ, et al. International Expert Consensus Document on takotsubo Syndrome (Part II): Diagnostic workup, outcome, and management. Eur Heart J.2018;39(22):2047-62.

24. Chiariello GA, Bruno P, Colizzi C, Crea F, Massetti M. takotsubo cardiomyopathy following cardiac surgery. J Card Surg. 2015;31(2):89-95.

25. Lyon AR, Bossone E, Schneider B, Sechtem U, Citro R, Underwood SR, et al. Current state of knowledge on takotsubo syndrome: a Position Statement from the Taskforce on takotsubo Syndrome of the Heart Fai- 
lure Association of the European Society of Cardiology. Eur J Heart Fail. 2015;18(1):8-27.

26. Angelini $P$, Monge J, Simpson L. Biventricular takotsubo cardiomyopathy: case report and general discussion. Tex Heart Inst J. 2013:40(3):3125.

27. Ehl NF, Zurek M, Rickli H, Maeder MT. "Double takotsubo": first description of the sequence of classical followed by inverted type in a young woman. Int J Cardiol. 2014;174(2):e367.

28. Medina de Chazal H, Del Buono MG, Keyser-Marcus L, Ma L, Moeller FG, Berrocal D, et al. Stress cardiomyopathy diagnosis and treatment. J Am Coll Cardiol. 2018;72(16):1955-71.

29. Rodríguez M, Rzechorzek W, Herzog E, Lüscher TF. Misconceptions and facts about takotsubo Syndrome. Am J Med. 2019;132(1):25-31.

30. Dawson DK. Acute stress-induced (takotsubo) cardiomyopathy. Heart. 2017;104(2):96-102.

31. Frangieh $\mathrm{AH}$, Obeid S, Ghadri J, Imori Y, D'Ascenzo F, Kovac M, et al. ECG Criteria to differentiate between takotsubo (Stress) cardiomyopathy and myocardial infarction. J Am Heart Assoc. 2016;5(6).e003418.

32. Kato K, Lyon AR, Ghadri JR, Templin C. takotsubo syndrome: aetiology, presentation and treatment. Heart. 2017;103(18):1461-9.

33. Watanabe M, Izumo M, Akashi YJ. Novel understanding of takotsubo Syndrome. Int Heart J. 2018;59(2):250-5.

34. Jaguszewski M, Osipova J, Ghadri JR, Napp LC, Widera C, Franke J, et al. A signature of circulating microRNAs differentiates takotsubo cardiomyopathy from acute myocardial infarction. Eur Heart J. 2013;5(15):999-1006.
35. Ghadri J-R, Cammann VL, Templin C. The International Takotsubo Registry: Rationale, design, objectives, and first results. Heart Fail Clin. 2016; 12(4):597-603.

36. Zhang L, Piña IL. Stress-induced cardiomyopathy. Heart Fail Clin. 2019; 15(1):41-53.

37. Napp LC. The risk of takotsubo Syndrome: Seeing the light. JACC Heart Fail. 2019;7(2):155-7.

38. Parodi G, Sanna GD. Trying to catch the octopus in the trap: takotsubo syndrome natural history. Int J Cardiol. 2018 Sep 15;267:48-9.

39. Taqueti VR, Di Carli MF. Coronary microvascular disease pathogenic mechanisms and therapeutic options. J Am Coll Cardiol. 2018;72(21): 2625-41.

40. Prasitlumkum N, Kittipibul V, Limpruttidham N, Rattanawong P, Chongsathidkiet $\mathrm{P}$, Boondarikpornpant $\mathrm{T}$. The presence of atrial fibrillation in takotsubo cardiomyopathy is predictive of mortality: Systematic review and meta-analysis. Ann Noninvasive Electrocardiol. 2018;24(1):e12566.

41. Pelliccia F, Pasceri V, Patti G, Tanzilli G, Speciale G, Gaudio C, et al. Long-term prognosis and outcome predictors in takotsubo Syndrome. JACC Heart Fail. 2019;7(2):143-54.

42. Scally C, Rudd A, Mezincescu A, Wilson H, Srivanasan J, Horgan G, et al. Persistent long-term structural, functional, and metabolic changes after stress-induced (takotsubo) cardiomyopathy. Circulation. 2018; 137(10):1039-48. 